\title{
Re-direction of carbon flux to key precursor malonyl-CoA via artificial small RNAs in photosynthetic Synechocystis sp. PCC 6803
}

\author{
Tao Sun 1,2,3, Shubin Li ${ }^{1,2,3}$, Xinyu Song ${ }^{1,4}$, Guangsheng Pei ${ }^{1,2,3}$, Jinjin Diao 1,2,3, Jinyu Cui 1,2,3, Mengliang Shi ${ }^{1,2,3}$,
} Lei Chen ${ }^{1,2,3}$ and Weiwen Zhang ${ }^{1,2,3,5^{*}}$

\begin{abstract}
Background: Photosynthetic cyanobacteria have attracted a significant attention as promising chassis to produce renewable fuels and chemicals due to their capability to utilizing solar energy and $\mathrm{CO}_{2}$. Notably, the enhancing supply of key precursors like malonyl-CoA would benefit the production of many bio-compounds. Nevertheless, the lacking of genetic tools in cyanobacteria, especially the knockdown strategies for essential pathways, has seriously restricted the attempts to re-direct carbon flux from the central carbohydrate metabolism to the synthesis of bioproducts.

Results: Aiming at developing new genetic tools, two small RNA regulatory tools are reported for the model cyanobacterium Synechocystis sp. PCC6803, based on paired termini RNAs as well as the exogenous Hfq chaperone and MicC scaffold ( $\mathrm{Hfq}-\mathrm{MiCC}$ ) previously developed in Escherichia coli. Both regulatory tools functioned well in regulating exogenous reporter gene lacZ and endogenous glgC gene in Synechocystis sp. PCC6803, achieving a downregulation of gene expression up to $90 \%$ compared with wildtype. In addition, the Hfq-MicC tool was developed to simultaneously regulate multiple genes related to essential fatty acids biosynthesis, which led to decreased fatty acids content by $11 \%$. Furthermore, aiming to re-direct the carbon flux, the Hfq-MicC tool was utilized to interfere the competing pathway of malonyl-CoA, achieving an increased intracellular malonyl-CoA abundance up to $41 \%$ ( 698.3 pg/mL/ $\mathrm{OD}_{730 \mathrm{~nm}}$ ) compared to the wildtype. Finally, the Hfq-MicC system was further modified into an inducible system based on the theophylline-inducible riboswitch.
\end{abstract}

Conclusions: In this study, two small RNA regulatory tools for manipulating essential metabolic pathways and redirecting carbon flux are reported for Synechocystis sp. PCC6803. The work introduces efficient and valuable metabolic regulatory strategies for photosynthetic cyanobacteria.

Keywords: Cyanobacteria, Metabolic regulation, Small RNA tools, Malonyl-CoA

\section{Background}

Environmental pollution and climate change resulted from over-consumption of fossil fuels have prompted the researches and development on green fuels and chemicals based on various types of "microbial cell factories" $[1,2]$. Among them, photosynthetic cyanobacteria have attracted significant attention as promising chassis for producing green fuels and chemicals due to their

\footnotetext{
*Correspondence: wwzhang8@tju.edu.cn

1 Laboratory of Synthetic Microbiology, School of Chemical Engineering \& Technology, Tianjin University, Tianjin 300072, People's Republic of China Full list of author information is available at the end of the article
}

capability to utilizing sunlight and $\mathrm{CO}_{2}$ as the sole energy and carbon sources, respectively [3]. Up to now, dozens of industrially relevant compounds have been successfully synthesized in several model cyanobacteria, such as Synechocystis sp. PCC 6803 (hereafter Synechocystis), Synechococcus elongatus PCC 7942, and Synechococcus sp. PCC 7002 [3].

Nevertheless, compared with other chassis microbes like Escherichia coli [4], lacking of genetic tools in cyanobacteria still seriously limits the development and application of cyanobacterial producing systems $[5,6]$. In $E$. coli, development and application of various genetic tools 
such as promoters, riboswitches, ribosome-binding site (RBS) libraries, CRISPR/Cas system (clustered regularly interspaced short palindromic repeats/CRISPR-associated proteins), and small RNA (sRNA) regulatory tools have made it easy to regulate both endogenous and exogenous metabolic pathways to achieve high productivity [7-10]. Among them, genetic tools based on artificial sRNA molecules have showed promising applications [11] as sRNA regulatory tools hardly imposed any metabolic burden on host cells [12]. In addition, the modularity, tunable base-pair complementation, and trans-acting ability of sRNA molecules allow for genome-wide regulation of target genes to achieve fine flux control $[10,13$, 14]. Moreover, the traditional deletion strategy of the essential genes or pathways is usually lethal to the host cell, while these genes or pathways could be knocked down via sRNA regulatory tools [15].

In a previous study with $E$. coli, Nakashima et al. [16] reported a sRNA tool based on paired termini antisense RNAs (named as PTRNAs). The tool contained $38 \mathrm{bp}$ flanking inverted repeats to stabilize the middle functioning antisense RNA (asRNA) designed based on the target gene (Fig. 1a), which was able to reach a $78 \%$ reduction of the acetate kinase-phosphotransacetylase operon in E. coli [16]. In addition, this strategy was later demonstrated effective in regulating various essential genes or pathways [15, 17]. Recently, Yang et al. [18] applied this strategy to regulate fatty acids biosynthesis, successfully
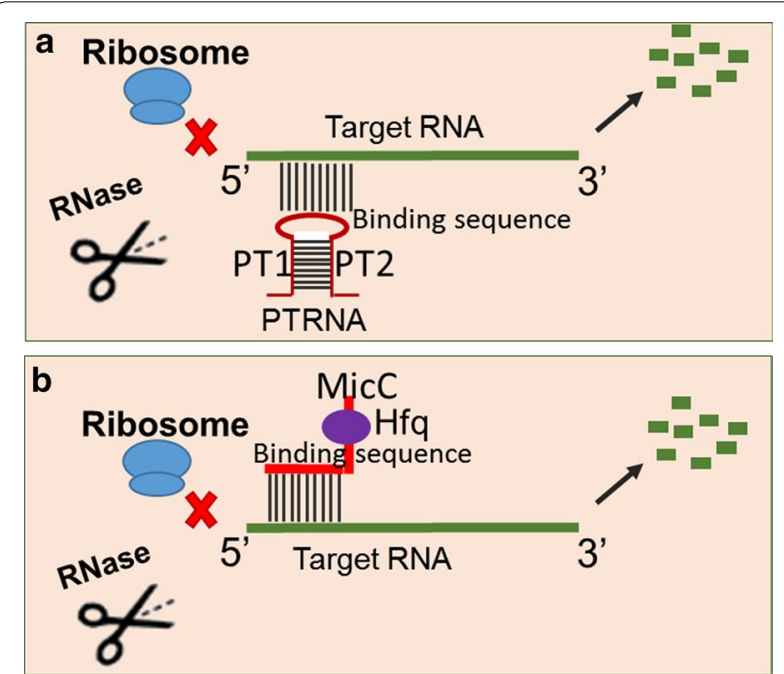

Fig. 1 Function and design principles for the PTRNA and Hfq-MicC tools. a Schematic of the PTRNA tool. The PTRNA contained a paired terminus composed of PT1 and PT2. The $100 \mathrm{bp}$ binding sequence in PTRNA was designed by choosing the antisense fragment from the translational starting site of the target gene. $\mathbf{b}$ Schematic of the $\mathrm{Hfa}$ $\mathrm{MicC}$ tool. The Hfq-MicC tool contained a 79 bp scaffold named MicC that could be recognized by the chaperone $\mathrm{Hfq}$. The $24 \mathrm{bp}$ binding sequence before MicC was designed by choosing the antisense fragment from the translational starting site of the target gene enhancing the supply of key intermediate malonyl-CoA and the production of malonyl-CoA-based derivate such as 4-hydroxycoumarin and resveratrol by 4.5-, 2.53-, and 1.70 -fold in E. coli, respectively. In another study, $\mathrm{Na}$ et al. [10] developed a new sRNA regulatory tool based on a chaperone protein $\mathrm{Hfq}$ and a well-studied sRNA scaffold from MicC in E. coli, in which the designed target-binding region was fused into $\mathrm{MicC}$ scaffold and the fused fragment could achieve effective regulation of target genes with the aid of Hfq chaperone (named as HfqMicC; Fig. 1b). The chaperone Hfq has three important roles including expediting annealing of sRNA to its target mRNAs, recruiting the major endo-ribonuclease RNase E for cleavage of the targets, and protecting the sRNAs from endonucleolytic cleavage [19-21]. More recently, the applicability of $\mathrm{Hfq}-\mathrm{MicC}$ tool was also demonstrated in Clostridium acetobutylicum [22].

Identification and characterization of cyanobacterial endogenous sRNAs were currently quite limited [23-25], which has restricted the development of sRNA regulatory tools directly using endogenous sRNAs. Up to now, a few studies have focused on the development of sRNAs regulatory tools in cyanobacteria. Recently, post-transcriptional regulatory tool based on E. coli IS10 RNA-IN/ OUT regulator was introduced into Synechococcus sp. PCC 7002 [26], even though inapplicability of this system in controlling endogenous genes makes it challenging for further application in cyanobacteria. To address this issue, in this study, we aim to extend the application of the PTRNA and Hfq-MicC tools in cyanobacteria. First, lac $Z$ encoding $\beta$-galactosidase and endogenous gene $g l g C$ related to glycogen biosynthesis were used as the reporter genes to evaluate functionality of PTRNA and Hfq-MicC systems in Synechocystis, respectively. In addition, Hfq-MicC tool was utilized to interfere multiple genes in the essential pathways related with fatty acid biosynthesis and malonyl-CoA generation in Synechocystis, achieving a re-direction of carbon flux and up to $41 \%$ increase $(\sim 698.3 \mathrm{pg} / \mathrm{mL} / \mathrm{OD})$ of intracellular malonylCoA abundance compared to wild type (WT). Moreover, the Hfq-MicC tool was modified to be an inducible system based on the theophylline-induced riboswitch. The study introduced efficient and valuable gene regulation strategies for metabolic engineering and synthetic biology in cyanobacteria.

\section{Results}

Constructing plasmids for gene expression in Synechocystis Aiming at constructing a vector that could replicate in E. coli and integrate into the genome of Synechocystis, the pTZ57R/T (Thermo Fisher Scientific Inc., CA, USA) was used as a backbone. Two homologous arms based on the neutral integration site of slr0168 [27], 
a chloramphenicol-resistant cassette (amplified from pACYC184; hereafter $\mathrm{Cm}^{\mathrm{R}}$ ), a strong promoter Pcpc560 [28] or the light-induced PpsbA2 [29], a multiple cloning site (MCS), and the terminator TrbcL [27] were obtained separately, and then successively ligated into backbone of pTZ57R/T, leading to the successful construction of pCP0168 (using Pcpc560) or pBA0168 (using PpsbA2), respectively (Additional file 1: Fig. S1A; detailed sequences were summarized in Additional file 2: Table S2). Both constructs were confirmed by Sanger sequencing.

\section{Constructing plasmids for sRNAs expression in Synechocystis}

For the construction of sRNA-expressing plasmids, the slr2030 and slr2031 genes were chosen as neutral integration sites [30]. Unlike pCP0168, a variant promoter named PpsbAM without native RBS sequence of PpsbA2 was chosen to prevent the possible translation of sRNAs and ensure the interaction between sRNA and its target mRNA [29]. In addition, a spectinomycin-resistant cassette (hereafter $\mathrm{Spe}^{\mathrm{R}}$ ) was utilized (amplified from pXT37b kindly provided by Prof. Xuefeng Lu) [27], leading to pBA3031M (Additional file 1: Fig. S1B).

For the construction of PTRNA-expressing plasmid, 38 bp of PT1 sequence (Additional file 2: Table S2) was introduced at a location after PpsbAM in pBA3031M via inverse PCR. The linear fragment was self-ligated using T4 DNA ligase (Thermo Fisher Scientific Inc., CA, USA), leading to pBA3031-PT (Fig. 2a; Additional file 2: Table $\mathrm{S} 2$ ). The $100 \mathrm{bp}$ binding sequence was obtained by amplifying the antisense fragment from the translational starting site of the target gene [16] (Fig. 1a). The PT2 sequence (Additional file 2: Table S2) was added into the end of the binding sequence via a PCR. The construction was confirmed by Sanger sequencing.
For the construction of Hfq-MicC-expressing plasmid, the Pcpc560- $h f q$-TrbcL cassette ( $h f q$ was amplified from genome of E. coli K12; Additional file 2: Table S2) was achieved by overlapping PCR and then introduced into pBA3031M at a location after TrbcL. In addition, the $79 \mathrm{bp}$ fragment of the scaffold from micC (synthesized; Additional file 2: Table S2) was introduced into a location after PpsbA2M by inverse PCR, leading to the pBA3031HM (Fig. 2b; Additional file 2: Table S2). The 24 bp binding sequence (Fig. 1b) was designed by choosing the antisense fragment from the translational starting site of the target gene and then introduced into pBA3031-HM through inverse PCR and self-ligation [10]. The construction was confirmed by Sanger sequencing.

\section{Functional validation of PTRNA and Hfq-MicC systems in Synechocystis using lacZ}

A widely used reporter gene lacZ (Additional file 2: Table S2) encoding $\beta$-galactosidase was ligated into both pCP0168 and pBA0168, and then introduced into WT, generating WT-LacZS and WT-LacZW, respectively (Table 1). Then, pBA3031-PTLacZ (using PTRNAs targeting lacZ) and pBA3031-HMlacZ (using Hfq-MicC targeting lac $Z$ ) were designed, constructed, and introduced into WT-LacZS and WT-LacZW, leading to WT-PTLacZS, WT-PTLacZW, WT-HMLacZS, and WTHMLacZW, respectively (Table 1 ). To evaluate whether PTRNA and Hfq-MicC systems were able to function in Synechocystis, activities of $\beta$-galactosidase were comparatively measured among samples (volume* $\mathrm{OD}_{730}$ ${ }_{\mathrm{nm}}=1$ ) of WT, WT-LacZS, WT-LacZW, WT-PTLacZS, WT-PTLacZW, WT-HMLacZS, and WT-HMLacZW. As illustrated in Fig. 3a, $\beta$-galactosidase activity was much lower in WT-LacZW than that in WT-LacZS due to the weaker strength of PpsbA2 than Pcpc560. Notably, compared to WT-LacZS, $\beta$-galactosidase activity
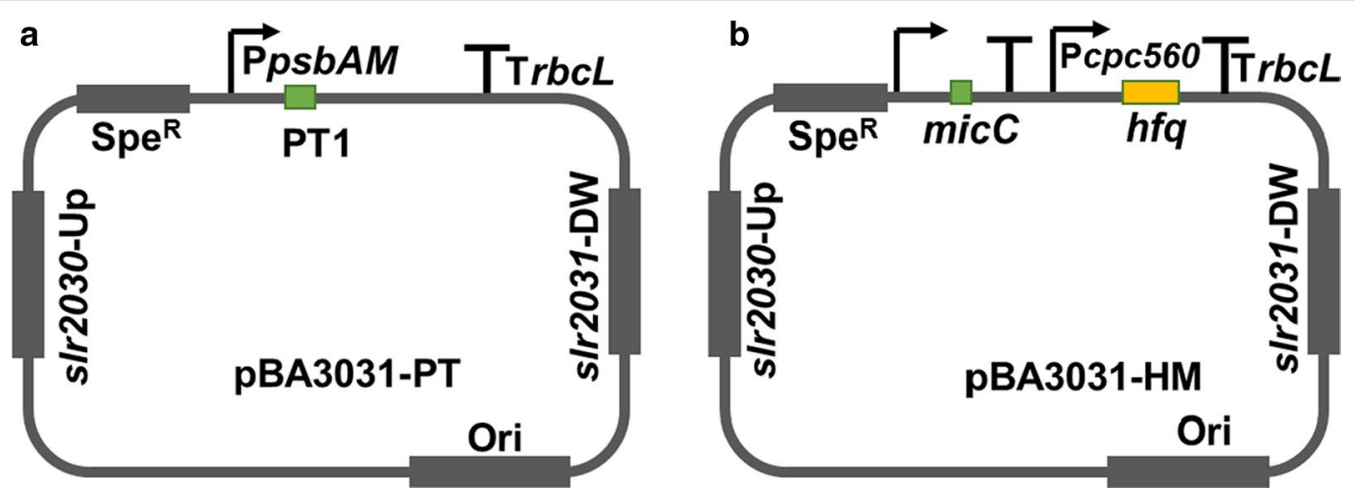

Fig. 2 Schematic of the constructed vectors for expressing sRNAs. Detailed sequences were available in Additional file 2: Table S2. a Schematic of the pBA3031-PT (Ori was the replicating element ColE1 essential for replication in E. coli.). b Schematic of the pBA3031-HM. The promoter and terminator for expressing sRNA was the same as the pBA3031-PT 
Table 1 Strains constructed in this study

\begin{tabular}{|c|c|}
\hline Strain & Genotype \\
\hline WT-LacZS & pCP0168::Pcpc560-lacZ-TrbcL; Cm in WT \\
\hline WT-LacZW & pCP0168::PpsbA2-lacZ-TrbcL; Cm ${ }^{R}$ in WT \\
\hline WT-PTLacZS & pBA3031 M::PpsbA2 M-PT1-aslacZ1-PT2-TrbcL; Spe ${ }^{R}$ in WT-LacZS \\
\hline WT-PTLacZW & pBA3031M::PpsbA2 M-PT1-aslacZ1-PT2-TrbcL; Spe in WT-LacZW \\
\hline WT-HMLacZS & pBA3031M::PpsbA2 M-aslacZ2-micC-TrbcL-Pcpc560-hfq-TrbcL; Spe in WT-LacZS \\
\hline WT-HMLacZW & pBA3031M::PpsbA2 M-aslacZ2-micC-TrbcL-Pcpc560-hfa-TrbcL; Spe ${ }^{R}$ in WT-LacZW \\
\hline WT-C & pBA3031M:: Spe ${ }^{R}$ in WT \\
\hline WT-PTGLGC & pBA3031M::PpsbA2 M-PT1-asglgC1-PT2-TrbcL; Spe ${ }^{R}$ in WT \\
\hline WT-HMC & pBA3031M::PpsbA2 M-asglgC2-micC-TrbcL; Spe ${ }^{R}$ in WT \\
\hline WT-HMGLGC & pBA3031M::PpsbA2 M-asglgC2-micC-TrbcL-Pcpc560-hfq-TrbcL; Spe ${ }^{R}$ in WT \\
\hline WT-HMFA & $\begin{array}{l}\text { pBA3031M::PpsbA2 M-ass/r1511-micC-TrbcL-PpsbA2 M-ass/r1332-micC-TrbcL-PpsbA2 M-ass/l1069-micC-TrbcL-Pcpc560-hfq-TrbcL; } \\
\text { Spe }{ }^{R} \text { in WT }\end{array}$ \\
\hline WT-HMMA1 & pBA3031M::PpsbA2 M-ass/r2023-micC-TrbcL-PpsbA2 M-ass/r1511-Pcpc560-hfq-TrbcL; Spe ${ }^{R}$ in WT \\
\hline WT-HMMA2 & $\begin{array}{l}\text { pBA3031M::PpsbA2 M-ass/r2023-micC-TrbcL-PpsbA2 M-ass/r1511-micC-TrbcL-PpsbA2 M-ass/r1332-micC-TrbcL-PpsbA2 M-ass//1069- } \\
\text { micC-TrbcL-Pcpc560-hfq-TrbcL; Spe }{ }^{R} \text { in WT }\end{array}$ \\
\hline WT-HMLacZS2 & pTR3031M::PpsbA2 M-aslacZ2-micC-TrbcL-Ptrc-riboswitch-hfa-TrbcL; Spe ${ }^{R}$ in WT-LacZS \\
\hline WT-HMMA1 $\left(\right.$ the $\left.^{*}\right)$ & pBA3031M::PpsbA2 M-ass/r2023-micC-TrbcL-PpsbA2 M-ass/r1511-Ptrc-riboswitch-hfq-TrbcL; Spe in WT \\
\hline
\end{tabular}

was decreased by $\sim 40$ and $\sim 47 \%$ in WT-PTLacZS and WT-HMLacZS, respectively; compared to WT-LacZW, $\beta$-galactosidase activity was, respectively, decreased by $~ 70$ and $72 \%$ in WT-PTLacZW and WT-HMLacZW (Fig. 3a). The results tentatively demonstrated the functionality of these two sRNA systems in regulating lacZ gene expression in Synechocystis.

\section{Functional validation of PTRNA and $\mathrm{Hfq}-\mathrm{MicC}$ tools in Synechocystis using $g l g C$}

Furthermore, we chose the native $g \operatorname{lgC}$ gene of Synechocystis involving glycogen biosynthesis to evaluate the functional performance of PTRNA and Hfq-MicC tools on regulating the endogenous gene. The plasmid pBA3031-PTGLGC and pBA3031-HMGLGC with the binding sequence targeting $g \lg C$ were designed and constructed based on PTRNA and Hfq-MicC tool, respectively, resulting in WT-PTGLGC and WT-HMGLGC, respectively (Table 1 ). Meanwhile, two strains, i.e., WT-C and WT-HMC, were constructed as controls (Table 1). WT-C obtained an empty vector of pBA3031M; thus, only the neutral site was replaced by $\mathrm{Spe}^{\mathrm{R}}$ compared to WT. In addition, WT-HMC only lacked the Pcpc560$h f q$-TrbcL cassette compared with WT-HMGLGC. The expression of sRNAs in WT-HMC, WT-PTGLGC and WT-HMGLGC was validated using RT-PCR (Additional file 1: Fig. S2). Consistent with the previous studies $[16,31]$, the expression level of sRNAs was more abundant in WT-PTGLGC and WT-HMGLGC than that in WT-HMC due to the protection by the paired termini or Hfq (Additional file 1: Fig. S2A). To investigate the performance of PTRNA and Hfq-MicC systems in regulating $g l g C$ gene expression, qRT-PCR assay (quantitative real-time polymerase chain reaction) was utilized for WT, WT-C, WT-HMC, WT-PTGLGC, and WTHMGLGC. As illustrated in Fig. 3b, the mRNA level of $g l g C$ in WT-PTGLGC was decreased by about $89 \%$ compared to that in WT, suggesting a very high efficiency of the PTRNA tool. In addition, without the chaperone Hfq, the mRNA level of $g \operatorname{lgC}$ in WT-HMC could also achieve a $20 \%$ decrease compared to that in WT (Fig. 3b), possibly due to the trans-regulatory role of sRNA alone. Notably, with the aid of Hfq, a $90 \%$ downregulation of $\operatorname{glgC}$ gene expression was observed in WT-HMGLGC compared to that in WT (Fig. 3b), indicating the functionality of Hfq-MicC tool in Synechocystis and the essential role of Hfq. Then, the glycogen quantitation assays were performed in the five strains including WT, WT-C, WTHMC, WT-PTGLGC, and WT-HMGLGC. As shown in Fig. 3c, though no significant difference in glycogen content was detected in WT-C and WT-HMC compared to WT, the glycogen content in WT-PTGLGC and WTHMGLGC was significantly decreased by 66.7 and $68.8 \%$ compared to WT at $72 \mathrm{~h}$. Finally, as it is well known that the $g l g C$-deficient strain had an obvious phenotype resistant to the bleaching process under nitrogen starvation [30, 32], nitrogen depletion assay was performed to verify the decrease of glycogen in WT-PTGLGC and WT-HMGLGC. As expected, both WT-PTGLGC and WT-HMGLGC showed a non-bleaching phenotype compared to WT (Fig. 3d). Though the non-bleaching phenotype was not completely inhibited in WT-PTGLGC and 

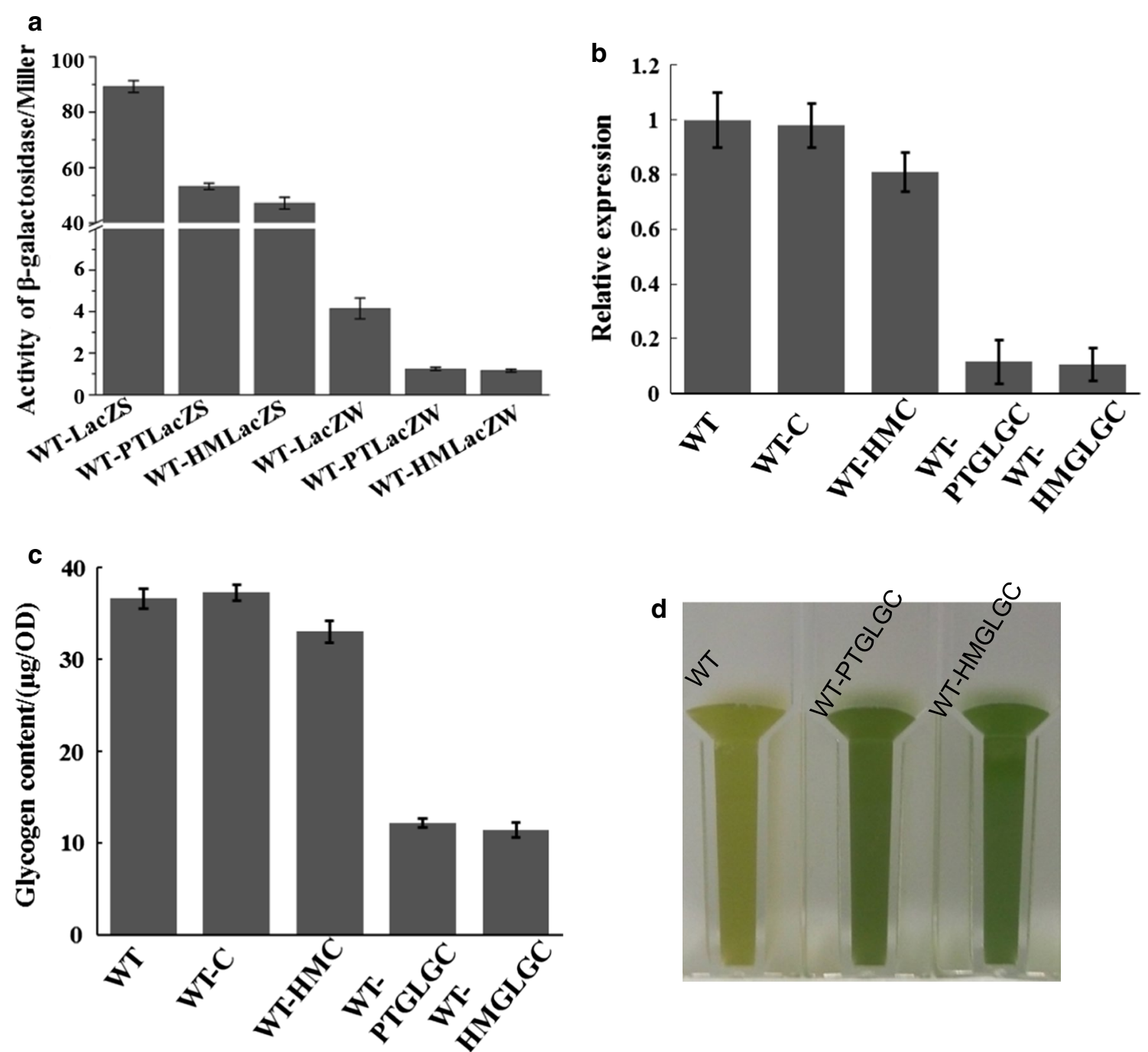

Fig. 3 Results of the $\beta$-Galactosidase activity measurement, qRT-PCR of glgC, glycogen quantitation and the nitrogen depletion assay. a $\beta$-Galactosidase activities in WT-LacZS, WT-LacZW, WT-PTLacZS, WT-PTLacZW, WT-HMLacZS, and WT-HMLacZW. The error bar represents the standard deviation of the three biological replicates for each sample. b qRT-PCR for the relative mRNA level of glgC in WT, WT-C, WT-HMC, WT-PTGLGC, and WT-HMGLGC. The error bar represents the standard deviation of the three technical replicates for each sample. c Glycogen quantitation assays in WT, WT-C, WT-HMC, WT-PTGLGC, and WT-HMGLGC. The error bar represents the standard deviation of the three biological replicates for each sample. $\mathbf{d}$ Nitrogen depletion assay in WT, WT-PTGLGC, and WT-HMGLGC

WT-HMGLGC, it could last for at least 2 days or more than 2 weeks under shaking or static culture condition, respectively. Altogether, based on both studies on lacZ and $g l g C$, the results showed that the Hfq-MicC tool was able to function better than the PTRNA tool in Synechocystis, as a higher percentage of downregulation of target gene was observed. Given its efficiency, the Hfq-MicC tool was chosen for the following application and optimization. In addition, as WT-C showed no difference from WT in the above assays, WT alone was thus used as the control for the following experiments.

\section{Knockdown of essential fatty acid biosynthesis pathway using $\mathrm{Hfq}-\mathrm{MicC}$ tool}

Utilizing the $\mathrm{Hfq}-\mathrm{MicC}$ tool established, we aimed at simultaneously regulating multiple genes of Synechocystis. The fatty acid biosynthetic pathway was selected, as it is essential and cannot be knocked out using the traditional manipulation strategy [18]. FabH encoding gene slr1511 related to initiation, and FabF encoding genes sll1069 and slr1332 related to elongation of fatty acid biosynthesis process were selected as target genes [33]. The WT-HMFA strain was constructed (Table 1; Fig. 5a), targeting sll1069, slr1332, and slr1511 genes involved in fatty acid biosynthesis (Fig. 4). The expression of sRNAs in WT-HMFA was 


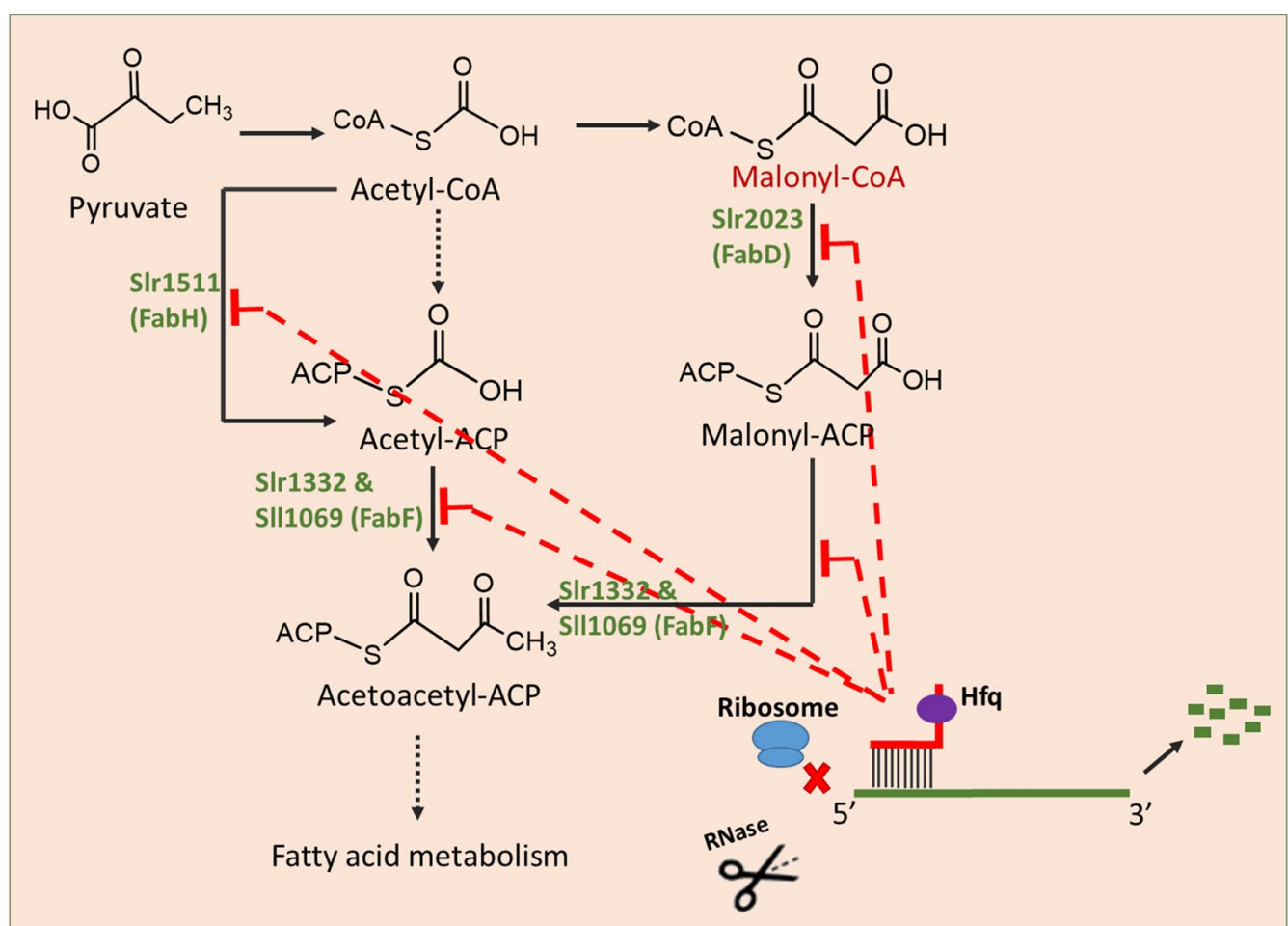

Fig. 4 Fatty acid biosynthesis, malonyl-CoA generation as well as the metabolic regulation strategy in Synechocystis. Fatty acid biosynthesis depends on the key precursor including acetyl-CoA and malonyl-CoA as well as the key enzymes like FabH, FabF, and FabD. Downregulation of the expression of the key genes using the Hfa-MicC tool would alter the generation of fatty acid and promote the accumulation of malonyl-CoA

validated using RT-PCR (reverse transcription polymerase chain reaction) (Additional file 1: Fig. S2). First, the qRT-PCR assay was carried out to determine the mRNA abundances of three target genes in WT-HMFA. As illustrated in Fig. 6a-c, transcriptional level of sll1069, slr1332, and slr1511 was, respectively, decreased by 41,28 , and $48 \%$ compared to that in WT. In addition, the lipid profiles between WT and WT-HMFA were comparatively measured using a GC analysis, and the results showed that most of the detected fatty acids showed a decreased peak area in WT-HMFA compared to that in WT, leading to approximately $11 \%$ decrease in total fatty acid content (Fig. 7a). Notably, C16:0, C16:1n7, C16:2n4, and C18:3n6 were significantly decreased by $14,18,25$, and $10 \%$ in WT-HMFA, respectively, compared with that in WT. Together, these results demonstrated functionality of the $\mathrm{Hfq}-\mathrm{MicC}$ tool in simultaneously regulating multiple genes of the fatty acid biosynthetic pathway in Synechocystis.

\section{Re-direction of carbon flux from fatty acid biosynthesis to key precursor malonyl-CoA}

Malonyl-CoA is an important precursor for many bioproducts like polyketides, flavonoids, 3-hydroxypropionic acid (3-HP), 4-hydroxycoumarin, tetracyclines, and doxorubicin [18, 34], and thus, the enhancing cellular pool of malonyl-CoA could potentially benefit the production for malonyl-CoA-derived products. The previous studies have suggested fatty acid biosynthesis as the major competitive pathway for malonyl-CoA generation [18]. To re-direct more carbon sources towards malonyl-CoA, we designed two other strains based on WT-HMFA. First, two genes, slr2023 (encoding FabD) and $\operatorname{sir} 1511$ (encoding FabH), were regulated by the HfqMicC tool in WT-HMMA1 as FabD and FabH were the key enzymes to catalyze the conversion of malonyl-CoA and its precursor acetyl-CoA into fatty acid synthesis, respectively (Table 1; Figs. 4 and 5b). Second, sll1069 and slr1332 putatively encoding FabF were also targeted with the Hfq-MicC tool along with slr1511 and slr2023 in WT-HMMA2 to decrease the consumption of both malonyl-CoA and its precursor acetyl-CoA (Table 1; Fig. 4 and $5 \mathrm{c}$ ). The expression of sRNAs in WT-HMMA1 and WT-HMMA2 was validated using RT-PCR (Additional file 1: Fig. S2). Similarly, the qRT-PCR was performed first to validate the knockdown effect of the Hfq-MicC tool on all four target genes. Notably, as much as 96 and 97\% mRNA of slr1511 and slr2023 were, respectively, downregulated in WT-HMMA1 (Fig. 6c, d), while 26, 


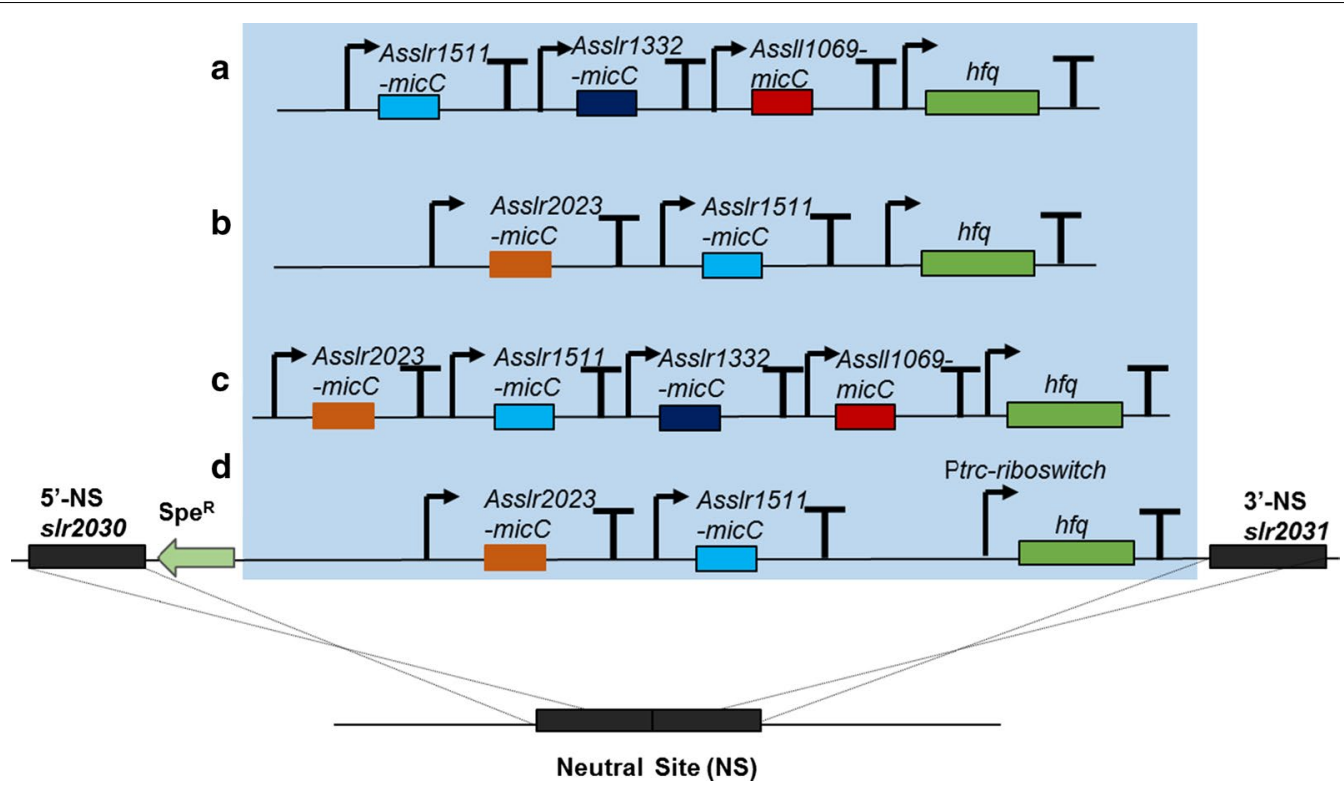

Fig. 5 Constructed strains using the Hfq-MicC tool. PpsbA2 M promoter was used for expressing all sRNAs, while Pcpc560 was utilized to express the chaperone $h f q$ except WT-HMMA1 (the*). TrbcL was used as the terminators. a Schematic of the WT-HMFA strain, in which s/r1511, s/r1322, and s/11069 were downregulated. b Schematic of the WT-HMMA1 strain, in which s/r2023 and s/r1511 were downregulated. c Schematic of the WT-HMMA2 strain, in which s/r2023, s/r1511, s/r1322, and s/l1069 were downregulated. d Schematic of the WT-HMMA1(the*) strain, in which s/r2023 and s/r1511 were downregulated. A modified promoter Ptrc contained a theophylline-responsive riboswitch was used to control the expression of $h f a$

49, 22, and 31\% decrease were measured for sll1069, slr1332, slr1511, and slr2023 in WT-HMMA2, respectively (Fig. 6). In addition, to determine the intracellular malonyl-CoA abundance, a bacterial malonyl-CoA quantitation kit was used for WT, WT-HMMA1, and WTHMMA2. Samples (volume* $\mathrm{OD}_{730 \mathrm{~nm}}=0.5$ ) for analysis were collected at middle-exponential phase $(48 \mathrm{~h})$ and end-exponential phase $(72 \mathrm{~h})$. The results showed a significant increase of malonyl-CoA content up to 69.11 and $69.83 \mathrm{pg} / \mathrm{mL}$ under all tested conditions in WT-HMMA2 for both 48 and $72 \mathrm{~h}$, indicating an enhancing supply of malonyl-CoA by $41 \%\left(\sim 691.1 \mathrm{pg} / \mathrm{mL} / \mathrm{OD}_{730 \mathrm{~nm}}\right)$ and $26 \%$ $\left(\sim 698.3 \mathrm{pg} / \mathrm{mL} / \mathrm{OD}_{730 \mathrm{~nm}}\right)$, respectively (Fig. 7b, c). Interestingly, although gene expression of slr1511 and slr2023 was almost fully blocked in WT-HMMA1, the malonylCoA content in WT-HMMA1 was only slightly increased by $9 \%$ (i.e., $22.96 \mathrm{pg} / \mathrm{mL}$; $229.6 \mathrm{pg} / \mathrm{mL} / \mathrm{OD}_{730 \mathrm{~nm}}$ ) at $72 \mathrm{~h}$ when compared with WT (Fig. 7c). Nevertheless, the results clearly demonstrated the application of the Hfq$\mathrm{MicC}$ tool in metabolic regulation for Synechocystis.

\section{Development of an inducible Hfq-MicC system}

Although regulation mediated by the sRNA tools has not totally blocked the expression of essential genes or pathways, the over downregulation could also affect cell growth and metabolism due to the essential roles played by the targets in supporting cell survival. Previously, growth inhibition was also observed in $E$. coli when PTRNA was used to achieve the knockdown of fabI related to fatty acid metabolism [16]. In this study, as slr1511 and slr2023 were almost fully blocked (up to 96 and $97 \%$, respectively) in HMMA-1, we also observed severe growth inhibition (Fig. 7d) and bleaching phenotype (Additional file 1: Fig. S5) for the strain, such "negative effects" could restrict our goal of improving malonyl-CoA accumulation in cyanobacterial chassis for its biotechnological application.

To address the issue, efforts can be made to make the Hfq-MicC system more controllable, so that normal biomass accumulation without interference is allowed at the early stage of cultivation, while malonyl-CoA accumulation is induced for biosynthesis of chemicals at the late stage of cultivation or anytime. Towards this goal, first, the promoter of Pcpc560-hfq-TrbcL cassette in pBA3031HM was replaced with the Ptrc containing a theophylline-induced riboswitch [35] (the new vector was named as pBA3031-HM(the*); Additional file 1: Fig. S6, Additional file 2: Table S2). Then, the binding sequence for lac $Z$ was ligated into pBA3031-HM(the $\left.{ }^{*}\right)$ and introduced into WT-LacZS, leading to WT-HMLacZS2 (Table 1). The $\beta$-galactosidase activity was comparatively measured among WT, WT-LacZS, and WT-HMLacZS2. As shown in Fig. 8a, $\beta$-galactosidase activity was decreased by $\sim 25 \%$ in WT-HMLacZS2 without induction. As a 

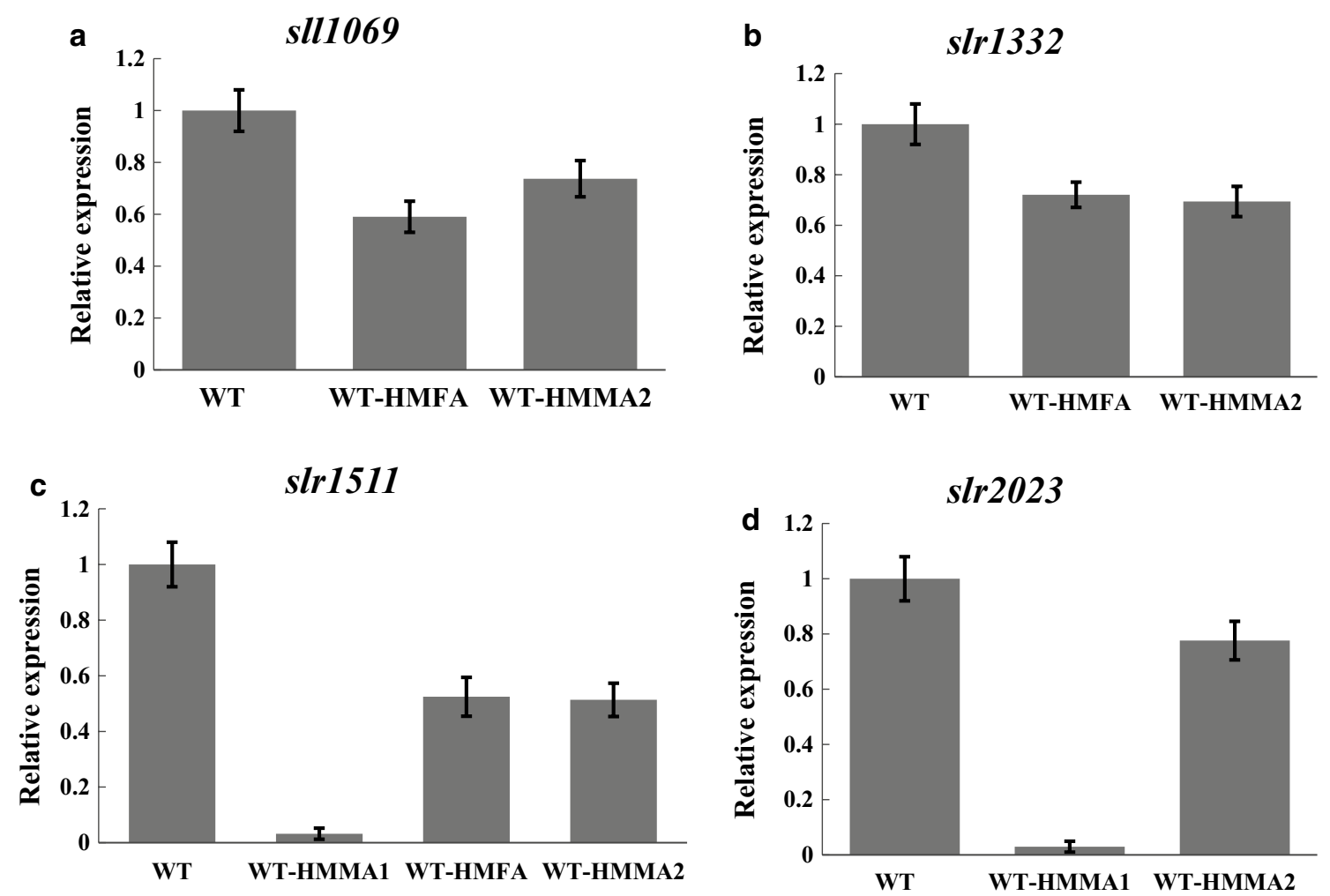

Fig. 6 qRT-PCR assays for detecting the mRNA levels. The error bar represents the standard deviation of the three technical replicates for each sample. a Relatively transcriptional level of s/1069 in WT, WT-HMFA, and WT-HMMA2. b Relatively transcriptional level of s/r1332 in WT, WT-HMFA, and WT-HMMA2. c Relatively transcriptional level of s/r1511 in WT, WT-HMFA, WT-HMMA1, and WT-HMMA2. d Relatively transcriptional level of s/r2023 in WT, WT-HMMA1, and WT-HMMA2

previous study has showed that the theophylline-induced riboswitch can tightly control protein expression in Synechocystis [36], the un-induced expression of Hfq can be neglected. Therefore, it was expected that the leakage in our system might be mostly due to the regulatory role of sRNA, as its binding sequence with 24 nucleotides complementary to the target mRNA could act as an antisense RNA [22]. After induction with $2 \mathrm{mM}$ theophylline (no toxicity of theophylline on the growth of WT at this concentration as shown in Additional file 1: Fig. S7) [36], the $\beta$-galactosidase activity was decreased by $\sim 52 \%$, suggesting the good performance of theophylline-induced riboswitch in controlling the translation of $h f q$. Based on the results using $l a c Z$, a modified strain of WT-HMMA1 named WT-HMMA1(the*) was constructed, in which the expression of $h f q$ was controlled by the theophyllineinduced riboswitch (Table 1; Fig. 5d). As expected, the growth inhibition observed previously was disappeared without induction before $48 \mathrm{~h}$ (Fig. 8b). After induction with $2 \mathrm{mM}$ theophylline, the slower growth of WTHMMA1(the*) than WT was observed at $72 \mathrm{~h}$ (Fig. 8b), which was in accordance with the inhibited growth of WT-HMMA1. Notably, an obvious $20 \%$ increase of
$43.86 \mathrm{pg} / \mathrm{mL}$ of malonyl-CoA content was obtained for WT-HMMA1(the*) compared to WT at $72 \mathrm{~h}$ (Fig. 8c) $\left(\sim 438.6 \mathrm{pg} / \mathrm{mL} / \mathrm{OD}_{730 \mathrm{~nm}}\right)$, which was much higher that found in the WT-HMMA1 containing a non-inducible system (i.e., 9\%). The results demonstrated the functionality of the inducible $\mathrm{Hfq}-\mathrm{MicC}$ system in regulating essential pathways in Synechocystis.

To evaluate the induction of Hfq, regulation efficiency as well as the potential leakage in WT-HMMA1(the*), sodium dodecyl sulfate polyacrylamide gel electrophoresis (SDS-PAGE), RT-PCR, and qRT-PCR assays were performed among WT and WT-HMMA1(the*) without or with induction. First, consistent with the previous report [36], the expression of Hfq was not detected in WTHMMA1 $\left(\right.$ the $^{*}$ ) without induction, while a significant signal could be obtained after $2 \mathrm{mM}$ theophylline induction (Additional file 1: Fig. S8). In addition, the expression level of Asslr1511-micC and Asslr2023-micC in WTHMMA1(the*) without induction was significantly lower than that in WT-HMMA1(the*) after induction due to the losing protection from Hfq (Fig. 8d). Moreover, as shown in Fig. 9a, nearly 80\% mRNA of both slr1511 and slr2023 was knocked down after induction. However, 


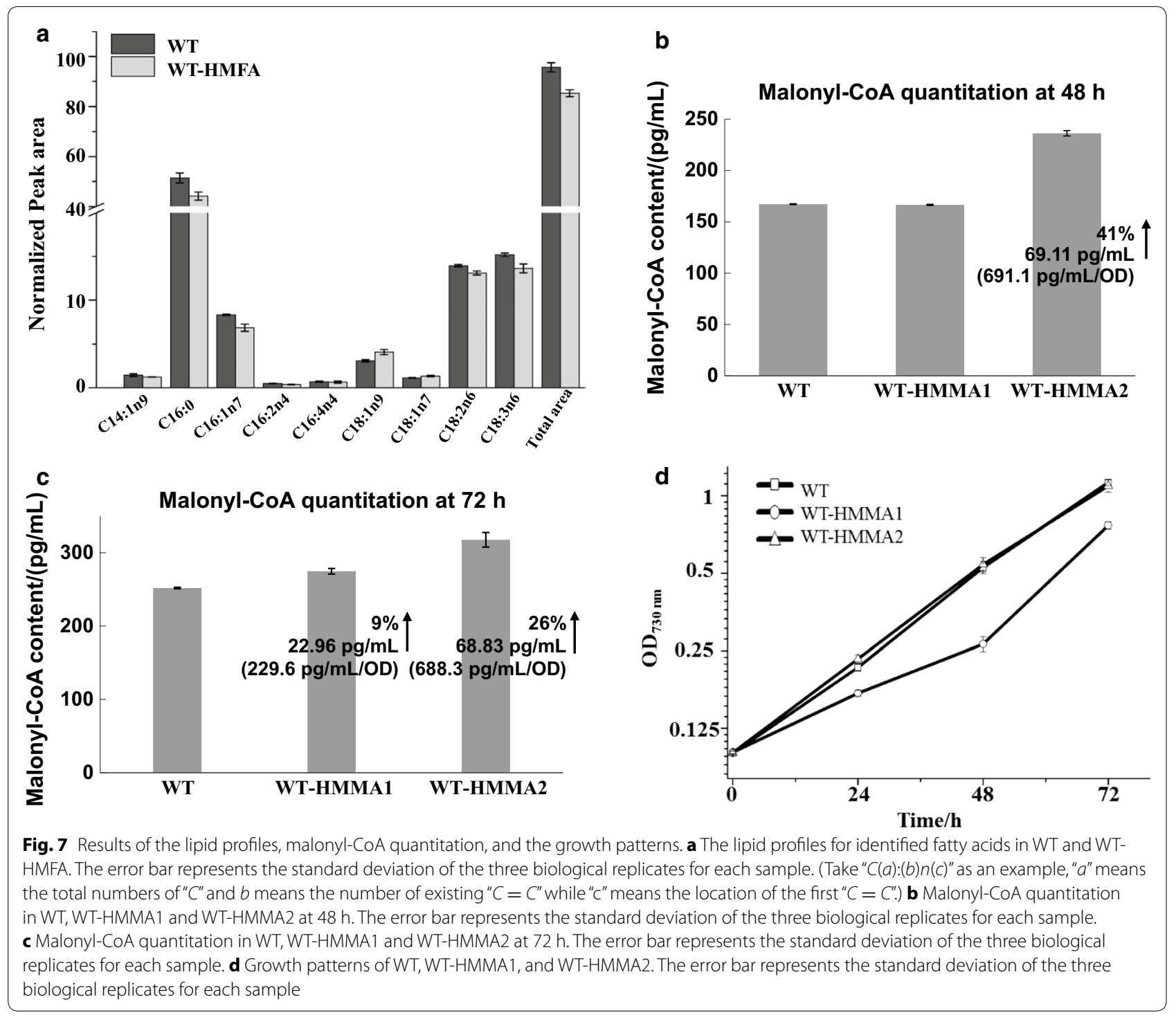

a $28 \%$ and $19 \%$ decrease were also observed for slr 1511 and slr2023 without induction, respectively, suggesting the possible leakage of the current system. As mentioned above, we suspected that the leakage was mainly from the role of sRNA without $h f q$. Thus, the lower of the binding energy between SRNA and its target, the higher of the leakage should be investigated. To evaluate this hypothesis, the IntaRNA tool developed for predicting interaction between sRNA and target gene for cyanobacteria was utilized [37, 38]. Consistently, the results showed that the binding energy was - $35.42 \mathrm{kcal} / \mathrm{mol}$ for $\operatorname{slr} 1511$ and its related sRNA, which was lower than that for slr2023 and its related sRNA (Fig. 9b; related sequences were listed in Additional file 2: Table S3). To make sure that the leakage problem was controllable, we further calculated the binding energy for all 3178 genes of the Synechocystis genome using their mRNA sequences and unique binding sRNAs for each gene. Interestingly, binding energy of most genes ( 82\%) was above $-35.42 \mathrm{kcal} / \mathrm{mol}$ (Additional file 1: Fig. S9), while only 3\% genes were found with a binding energy less than $-40 \mathrm{kcal} / \mathrm{mol}$ (Additional file 1: Fig. S9). The analysis results implied that the leakage could be controlled below $30 \%$ for most of the target genes in the Synechocystis genome using pBA3031-HM(the $\left.\mathrm{e}^{*}\right)$ under our tested conditions.

\section{Discussion}

In recent years, various genetic tools have gradually become available in cyanobacteria [26, 28, 30, 35, 39]. Nevertheless, inherent defects of the current genetic tools sometimes made it not suitable for metabolic engineering and synthetic biology. The expression levels of noninherent heterologous genes cloned in plasmid vectors can be tuned quite efficiently using these traditional tools 

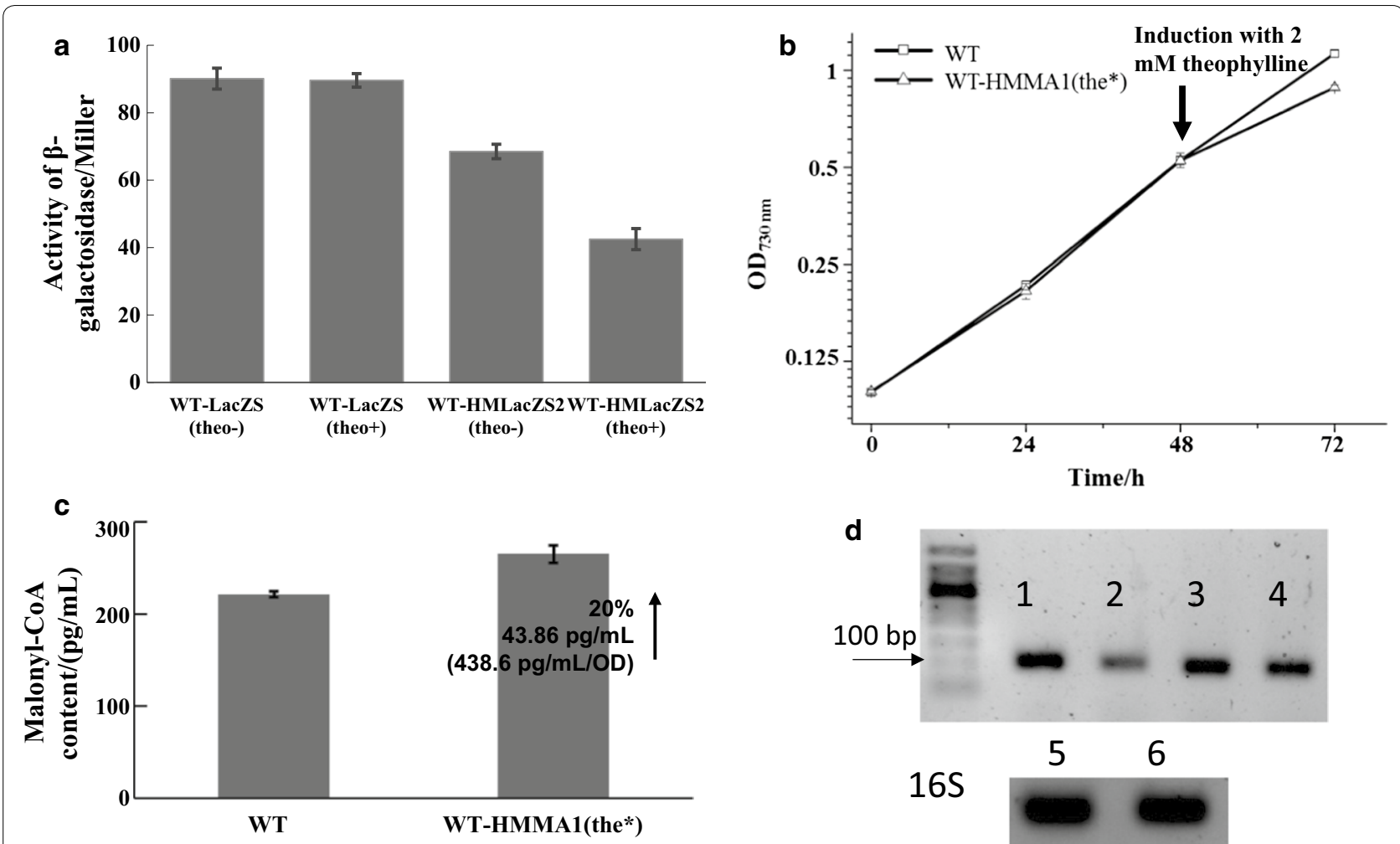

Fig. 8 Results of the $\beta$-galactosidase activity measurement, growth patterns, malonyl-CoA quantitation, RT-PCR. a $\beta$-Galactosidase activities in WTLacZS and WT-HMLacZS without (shown as "theo-") or with (shown as "theo+") 2 mM theophylline induction. The error bar represents the standard deviation of the three biological replicates for each sample. b Growth patterns for WT and WT-HMMA1 (the*). Both WT and WT-HMMA1 (the*) were induced with $2 \mathrm{mM}$ theophylline at $48 \mathrm{~h}$. The error bar represents the standard deviation of the three biological replicates for each sample. c Malonyl-CoA contents of WT and WT-HMMA1 (the*) at $72 \mathrm{~h}$. The error bar represents the standard deviation of the three biological replicates for each sample. d RT-PCR for WT-HMMA1 (the*) with or without induction. Lane 1-6:1 and 3 were the expression level of Ass/r1511-micC and Ass/r2023micC with $2 \mathrm{mM}$ theophylline induction, respectively; 2 and 4 were the expression level of Ass/r1511-micC and Ass/r2023-micC without induction, respectively; 4 and 6 were the expression level of $16 \mathrm{~S}$ rRNA in WT-HMMA1(the*) with or without induction, respectively

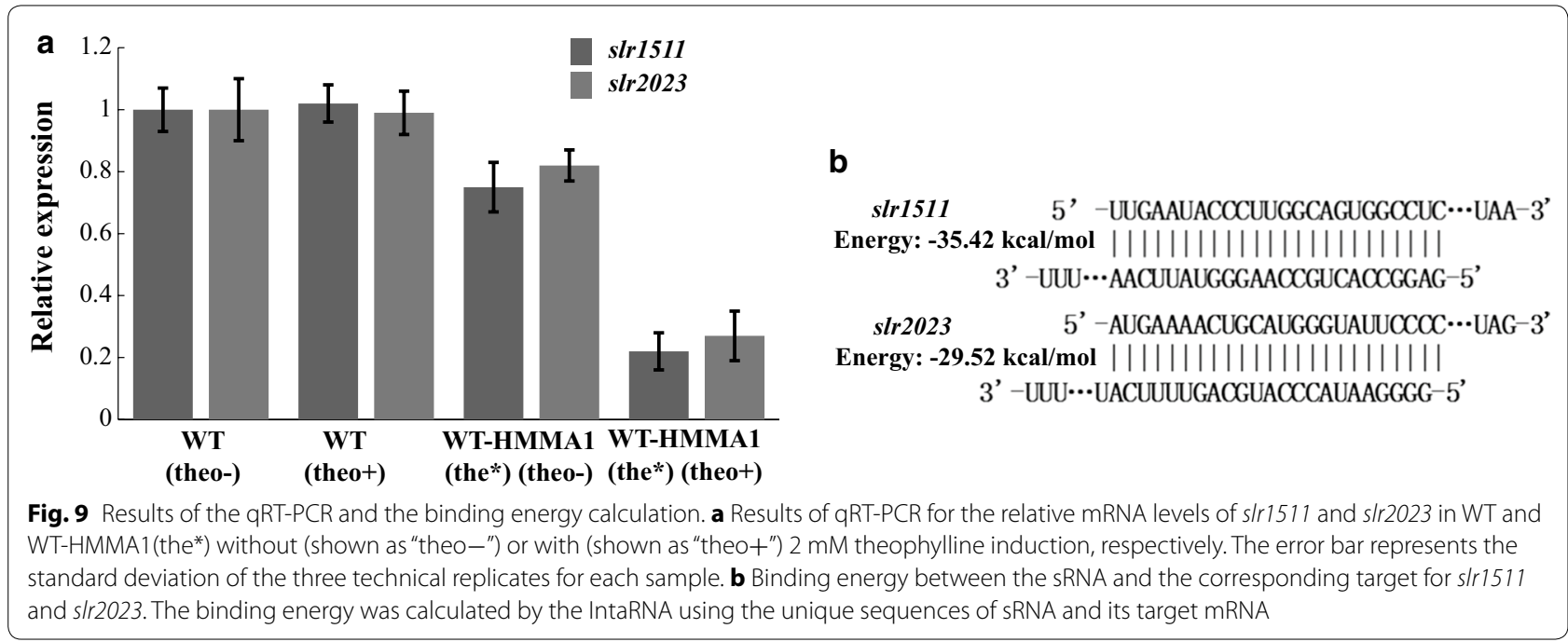


and methods like promoters, RBS sequences, and riboswitches, but those of inherent chromosomal genes are still laborious and time-consuming to manipulate [22]. In addition, transcriptional blockage of essential genes or pathways mediated by CRISPR/Cas system could be fatal to cyanobacteria [40]. In this study, aiming to extend the cyanobacterial genetic tool libraries, we developed two sRNA regulatory tools for the model cyanobacterium Synechocystis, based on the PTRNA and Hfq-MicC tools of $E$. coli, respectively. The results demonstrated their utilization in regulating single or multiple gene(s) in Synechocystis. Compared to other genetic tools available in cyanobacteria, the PTRNA and the Hfq-MicC tool are suitable for regulating both endogenous and exogenous gene(s), especially metabolically essential gene(s).

Primary role of fatty acids is to act as the hydrophobic component of the membrane lipids in both eukaryotes and prokaryotes [33]. Fatty acid synthases are responsible for the biosynthesis of fatty acids, extending its length by two carbon units every cycle [41]. Meanwhile, some of the intermediate metabolites are important precursors for synthesis of many bioproducts [3]. However, as fatty acid biosynthesis could compete carbon flux with synthesis of these bioproducts, it is necessary to develop technologies to regulate the essential fatty acid pathways, so that more carbon flux can be directed to bioproducts. Previously, Yang et al. [18] utilized PTRNAs to regulate the key genes $f a b D, f a b B, f a b F$, and $f a b H$ involving fatty acids biosynthesis in $E$. coli, successfully decreasing the fatty acid generation, and enhancing the supply of malonyl-CoA up to fivefold. However, due to the lack of suitable genetic tools for knocking down essential genes, regulation of fatty acid biosynthesis can hardly be performed in cyanobacteria. In cyanobacteria, FabH was claimed as the sole rate-limiting enzyme, and the ketosynthase FabF was found to be essential for fatty acid synthase turnover $[42,43]$. In accordance to these early results, interference of FabH (encoded by slr1511) and FabF (encoded by sll1069 and slr1332) in WT-HMFA by sRNA tools developed in this study led to decreased contents of fatty acids than WT. In addition, as demonstrated by the qRT-PCR analysis, the Hfq-MicC tool was efficient to interfere either one (WT-HMGLGC, Fig. 3b) or two targets (WT-HMMA1, Fig. 6c, d), with greater than $90 \%$ of gene expression decreased. It worth noting that with the number of target genes increased to three (in WTHMFA) or four (in WT-HMMA2), the interference efficiency of the Hfq-MicC tool was declining (Fig. 6). As the chaperone Hfq is essential for the Hfq-MicC system, one possible explanation for the declined efficiency with more target genes (i.e., > 3) could be due to the insufficient supply of Hfq chaperone, which was in consistent with the overexpression of synthetic sRNAs resulting in reduction of available $\mathrm{Hfq}$ proteins in E. coli [44]. In addition, another possible explanation could be due to the different expression levels of sRNAs in different constructed strains, although no significant differences could be seen from the RT-PCR assays (Additional file 1: Fig. S2B). Nevertheless, to validate these assumptions, in the future, the expression level of Hfq or sRNAs should be enhanced using stronger promoters or duplicate copies when regulating multiple genes to compare with the current one.

Malonyl-CoA served as a major building block for a variety of relevant derivatives like polyketides, flavonoids, and fatty acid-derived products [45]. As the generation of malonyl-CoA from acetyl-CoA was catalyzed by acetyl-CoA carboxylase (ACC) [45], manipulation of ACC has previously been demonstrated to be an effective strategy to enhance the malonyl-CoA pool in cells [46]. In our previous study, overexpression of four key genes encoding AccA, AccB, AccC, and AccD was also able to improve the malonyl-CoA supply, eventually leading to an increased 3-HP production up to 4.5-fold [34]. In addition, by engineering phospholipid synthesis transcriptional regulators in S. cerevisiae, a significant increase of malonyl-CoA content in the engineered strains and ninefold increase of the titer of malonylCoA-derived 3-HP were achieved [47]. In this work, in accordance with the previous study using PTRNA tool to regulate the malonyl-CoA supply [18], we decreased the flux from both acetyl-CoA and malonyl-CoA to fatty acids biosynthesis using the $\mathrm{Hfq}-\mathrm{MicC}$ tool to downregulating two (slr1511 and slr2023) or four genes (slr1511, sll1069, slr1332 and slr2023), leading to a significant increase of malonyl-CoA content in cells (Fig. 7b, c). The results provided a new strategy for increasing malonyl-CoA in cyanobacterial chassis for producing malonyl-CoA-derived chemicals. In addition, a recent study demonstrated the importance of the fine-tuning for gene expression, in which the highest production of putrescine in $E$. coli was not observed in strains with the greatest knockdown of the $\arg F$ gene expression, as the over downregulation of $\arg F$ could result in growth inhibition [44]. Consistently, though slr1511 and slr2023 were almost fully blocked in WT-HMMA1, the enhancement of malonyl-CoA was not significant as the growth of WTHMMA1 was also affected. On the contrary, though the four genes in WT-HMMA2 were only partially downregulated, a more significant increase of malonyl-CoA content was observed. Moreover, when modification of WT-HMMA1 to WT-HMMA1(the*) to allow biomass accumulation at the early stage was adopted, a higher increase (20\%) on malonyl-CoA was achieved (Fig. 8c), suggesting the importance of fine-tuning and potential value of sRNA regulatory tools. 
Recently, Gordon et al. [40] modified the CRISPR/ dCas9 systems in Synechococcus sp. PCC 7002 by introducing the TetR repressor and the aTc-responsive promoter to control the dCas9 expression. Combined with weak RBS for dCas9 and a weak promoter for both sgRNA and dCas9, the modified system achieved a finely tuning regulation of the target gene with a downregulated efficiency up to $90 \%$ [40]. Nevertheless, this modified system still carried a $~ 30 \%$ leakage without induction [40]. The synthetic theophylline-dependent riboswitch has been screened and optimized in the early studies $[48,49]$. A modified theophylline-dependent riboswitch was first introduced into cyanobacterium Synechococcus elongatus PCC 7942 in 2013 [35]. The transcript with this riboswitch could expose or sequester the RBS with or without the ligand theophylline, respectively, resulting in a negligible leakage and a significant induction up to 190-fold [35]. In this study, the theophylline-dependent riboswitch was utilized to control the expression of the chaperone protein $\mathrm{Hfq}$ and achieve a good performance of HfqMicC tool after induction, even though, 20-30\% leakage (depending on the binding energy between sRNA and its target) was still observed as the artificial sRNAs with 24 nucleotides complementary to the target mRNA could act as an antisense RNA. By fusing the theophylline-sensing aptamer to a spacer element followed by an aptamer-complementary sequence, the transcriptionregulating riboswitch has been found working in $E$. coli [50]. To decrease the leakage, transcriptional regulating riboswitch could be utilized in the future to accurately control the expression of sRNAs.

\section{Conclusion}

In this study, two small RNA regulatory tools are reported for Synechocystis based on PTRNAs and Hfq$\mathrm{MicC}$ previously developed in E. coli. The results demonstrated that both regulatory tools were well functioning in Synechocystis. In addition, the Hfq-MicC tool was further developed to simultaneously regulate multiple genes related to essential fatty acids biosynthesis and generation of the key precursor malonyl-CoA. Moreover, the $\mathrm{Hfq}-\mathrm{MicC}$ system was further modified to be an inducible system based on the theophylline-inducible riboswitch, achieving an optimized sRNA tool with good induction capability and relatively low leakage. The work introduces stable and efficient metabolic regulation strategies for photosynthetic cyanobacteria.

\section{Methods}

\section{Bacterial growth conditions}

WT and all Synechocystis construction strains were grown on BG-11 agar plate or in medium ( $\mathrm{pH} 7.5)$ under a light intensity of approximately $50 \mu \mathrm{moL}$ photons $\mathrm{m}^{-2} \mathrm{~s}^{-1}$ in an illuminating shaking incubator (HNY-211B, Honour, Tianjin, China) at $130 \mathrm{rpm}$ at $30^{\circ} \mathrm{C}$ or incubator (SPX-250B-G, Boxun, Shanghai, China) at $30{ }^{\circ} \mathrm{C}$, respectively [51]. Media for constructed strains were supplemented with appropriate antibiotic(s) when necessary (i.e., $10 \mu \mathrm{g} / \mathrm{mL}$ chloramphenicol or/and $10 \mu \mathrm{g} / \mathrm{mL}$ spectinomycin). E. coli strains were grown on LB agar plate or in LB medium with appropriate antibiotic(s) to maintain plasmids (i.e., $30 \mu \mathrm{g} / \mathrm{mL}$ chloramphenicol or $100 \mu \mathrm{g} / \mathrm{mL}$ spectinomycin) at $37{ }^{\circ} \mathrm{C}$ using incubator or shaking incubator (HNY-100B, Honour, Tianjin, China) at $200 \mathrm{rpm}$, respectively. Cell growth of Synechocystis was measured at $\mathrm{OD}_{730 \mathrm{~nm}}$ using a UV-1750 spectrophotometer (Shimadzu, Kyoto, Japan).

\section{Strain construction}

E. coli DH5 $\alpha$ strain was used for vector amplification and construction generation. All the primers were synthesized by GENEWIZ Inc. (Suzhou, China). Primers used in this study were listed in Additional file 2: Table S1 and the related sequences of cassettes used in this study were listed in Additional file 2: Table S2. All the strains constructed in this study were listed in Table 1 . Transformation of Synechocystis was performed by electroporation $(\sim 10 \mu \mathrm{g}$ plasmid DNA) using GenePulser Xcell (Bio-Rad, CA, USA) and grown photo-autotrophically on agar plate with $10 \mu \mathrm{g} / \mathrm{mL}$ spectinomycin and/or $10 \mu \mathrm{g} / \mathrm{mL}$ chloramphenicol [24]. All transformants were validated by colony PCR.

\section{$\beta$-Galactosidase activity}

A total of $1 \mathrm{~mL}$ of Synechocystis $\left(\mathrm{OD}_{730 \mathrm{~nm}}=1\right)$ culture were collected via centrifugation at $13,000 \times g$ and re-suspended using $1 \mathrm{~mL} \mathrm{Z} \mathrm{Buffer}\left(60 \mathrm{mM} \mathrm{Na}_{2} \mathrm{HPO}_{4}\right.$, $40 \mathrm{mM} \mathrm{NaH}_{2} \mathrm{PO}_{4}, 10 \mathrm{mM} \mathrm{KCl}, 1 \mathrm{mM} \mathrm{MgSO}$ and $40 \mathrm{mM}$ $\beta$-mercaptoethanol). $50 \mu \mathrm{L} 0.1 \% \mathrm{SDS}, 50 \mu \mathrm{L}$ chloroform, and $200 \mu \mathrm{L}$ ortho-nitrophenyl-beta-D-galactopyranoside (ONPG; $4 \mathrm{mg} / \mathrm{mL}$ ) were then added sequentially. The mixing solution was incubated at $30^{\circ} \mathrm{C}$ for $20 \mathrm{~min}$. Finally, $500 \mu \mathrm{L} 1 \mathrm{M} \mathrm{Na}_{2} \mathrm{CO}_{3}$ was added to stop the reaction. The final solution was centrifuged for $2 \mathrm{~min}$ at $13,000 \times g$ and the supernatant was measured at $\mathrm{OD}_{420 \mathrm{~nm}}$ using the UV-1750 spectrophotometer (Shimadzu, Kyoto, Japan). The results were normalized using the absorption of WT at $\mathrm{OD}_{420 \mathrm{~nm}}$. LacZ activity was quantized using the formula "Miller $=1000 \times \mathrm{OD}_{420 \mathrm{~nm}} /\left(1 \mathrm{~mL} \times 20 \mathrm{~min} \times \mathrm{OD}_{730 \mathrm{~nm}}\right) "$ according to a previous publication [52].

\section{Glycogen quantitation assays}

Glycogen quantitation assays were performed using a glycogen quantitation Kit (Tiangen, Beijing, China) and modified based on the previous studies [32, 53]. Briefly, $1 \mathrm{~mL}$ fresh cultures of WT, WT-C, WT-HMC, 
WT-PTGLGC, and WT-HMGLGC (Volume* ${ }^{\circ} D_{730}$ $\mathrm{nm}=1)$ were collected at $72 \mathrm{~h}$ by centrifugation at $14,000 \times g$ for $2 \mathrm{~min}$. Then, the sample pellets were collected and re-suspended with $500 \mu \mathrm{L} \mathrm{KOH}$ solution $(30 \% ; w / v)$, and then incubated for $2 \mathrm{~h}$ at $95{ }^{\circ} \mathrm{C}$. After that, $1500 \mu \mathrm{L}$ of absolute ethanol was added into the sample and incubated for $2 \mathrm{~h}$ on ice. The sample was centrifuged at $14,000 \times g$ for $10 \mathrm{~min}$ and the precipitate was re-suspended using $500 \mu \mathrm{L}$ of $\mathrm{ddH}_{2} \mathrm{O}$ and then incubated at $95{ }^{\circ} \mathrm{C}$ for $15 \mathrm{~min}$. The sample was then cooled on ice and added with anthrone $/ \mathrm{H}_{2} \mathrm{SO}_{4}$ solution $(w / v=2: 1)$ and incubated at $95^{\circ} \mathrm{C}$ for $15 \mathrm{~min}$. The final solution was cooled on ice and measured at $\mathrm{OD}_{625 \mathrm{~nm}}$. The quantitation of glycogen was performed by a standard curve using a series concentration of glucose solution (Additional file 1: Fig. S3). The absorption at $\mathrm{OD}_{625}$ $\mathrm{nm}$ by $111 \mu \mathrm{g}$ glycogen was equal to that by $100 \mu \mathrm{g}$ glucose according to the protocol.

\section{Nitrogen depletion assay}

BG-11 medium without sodium nitrate $\left(B G-N^{-}\right)$was used for the nitrogen depletion experiments [54]. Briefly, $5 \mathrm{~mL}$ fresh cultures of WT, WT-PTGLGC, and WT-HMGLGC $\left(\mathrm{OD}_{730 \mathrm{~nm}}=0.5\right)$ were collected by centrifugation at $3000 \times g$ and $4{ }^{\circ} \mathrm{C}$, washed twice using BG- ${ }^{-}$medium, and then inoculated into $25 \mathrm{~mL}$ of BG-N ${ }^{-}$liquid medium in $100 \mathrm{~mL}$ flasks. The assays were performed both in an illuminating shaking incubator or incubator for a shaking or static culture and repeated for 3 times to ensure the phenotype.

\section{qRT-PCR analysis}

Total RNA extraction was achieved through a Directzol $^{\text {TM }}$ RNA MiniPrep Kit (Zymo, CA, USA). Synechocystis samples were collected at $48 \mathrm{~h}$. Approximately $10 \mathrm{~mL}$ of samples $\left(\mathrm{OD}_{730 \mathrm{~nm}}=0.5\right)$ were collected by centrifugation $(7000 \times g$ for $10 \mathrm{~min})$, frozen immediately by liquid nitrogen after centrifugation, and subjected to RNA extraction following the manufacturer's protocol. cDNAs were synthesized using RevertAid First Strand cDNA Synthesis Kit following the manufacturer's protocol (Thermo Fisher Scientific Inc., CA, USA), and then, $1 \mu \mathrm{L}$ of each dilution was used as template for following qRT-PCR reaction. The qPCR reaction was carried out in $10 \mu \mathrm{L}$ reactions containing $5 \mu \mathrm{L}$ of UltraSYBR Mixture

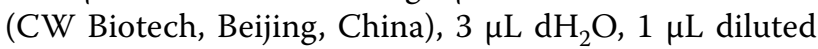
template cDNA, and $1 \mu \mathrm{L}$ of each PCR primer, employing the StepOnePlus ${ }^{\mathrm{TM}}$ Real-Time PCR System (Applied Biosystems, CA, USA) [24]. Three technical replicates were performed for each condition. Data analysis was carried out using the StepOnePlus analytical software (Applied Biosystems, CA, USA) and the $2^{-\Delta \Delta C T}$ method [55]. 16S rRNA was selected as a reference gene and the data were presented as ratios of the amount of normalized transcript in the constructed strain to that from the WT control [24].

\section{RT-PCR analysis}

The RNA extraction and cDNA synthesis were the same as described above. Then, specific forward primers of each sRNAs and the reverse primer micC-R were used to amplify each sRNAs (Primers were listed in Additional file 2: Table S1). 16S rRNA was selected as a reference.

\section{Lipid profiles}

Total lipids were extracted from approximate $5 \mathrm{mg}$ dry cells of the WT and WT-HMFA strain in $2 \mathrm{~mL}$ chloroform/methanol $(v / v, 2: 1)$ (each with 3 biological replicates) using ultrasonic treatment for $10 \mathrm{~min}$ and centrifugation at $7000 \times g$ for $5 \mathrm{~min}$. Then, $5 \mathrm{~mL} 2.0 \%$ $\mathrm{H}_{2} \mathrm{SO}_{4}$-methanol $\left(v / v, \mathrm{H}_{2} \mathrm{SO}_{4} /\right.$ methanol) was added into the collected supernatants, and the flask was stirred at $70{ }^{\circ} \mathrm{C}$ for $1 \mathrm{~h}$. After $2 \mathrm{~mL}$ of hexane and $0.75 \mathrm{~mL}$ of distilled water were added to the flask and mixed, the hexane layer contained the fatty acid methyl esters (FAMEs) was transferred to a new vial and mixed with the internal standard C17-ME for gas chromatography (GC) analyses. FAME analyses were carried out by an Agilent 6890 GC instrument (CA, USA) and FAME yield was calculated using the equation described previously by Liu et al. [56].

\section{Malonyl-CoA quantitation}

Quantitation of malonyl-CoA was performed using a "Bacterial Malonyl-CoA ELISA Kit" following the protocols (Dongge Biotech Inc., Beijing, China). Briefly, $1 \mathrm{~mL}$ of Synechocystis $\left(\mathrm{OD}_{730 \mathrm{~nm}}=0.5\right)$ culture was collected by centrifugation at $4{ }^{\circ} \mathrm{C}(13,000 \times g, 2 \mathrm{~min})$. Cell pellets

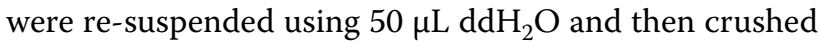
by multi-gelation with liquid nitrogen. After that, $10 \mu \mathrm{L}$ supernatant and $40 \mu \mathrm{L}$ diluent buffer were added into a 96-well plate. Then, $50 \mu \mathrm{L}$ of HRP-conjugate and $50 \mu \mathrm{L}$ of antibody were added into each well. The mixture was incubated under $37^{\circ} \mathrm{C}$ for $1 \mathrm{~h}$ and then washed five times using the wash buffer. After that, $50 \mu \mathrm{L}$ of Substrate A and $50 \mu \mathrm{L}$ of Substrate B were added and then incubated for $15 \mathrm{~min}$ at $37{ }^{\circ} \mathrm{C}$. Finally, the reaction was stopped by adding $50 \mu \mathrm{L}$ stop solution and measured at $\mathrm{OD}_{450}$ $\mathrm{nm}$ using the ELx808 Absorbance Microplate Reader (BioTek, Winooski, VT, USA). The standard curve was plotted using different dilutions of malonyl-CoA standard (Dongge Biotech Inc., Beijing, China) (Additional file 1: Fig. S1).

\section{Theophylline treatment}

The stock solution of theophylline was prepared by dissolving $10 \mathrm{mmol}$ theophylline (Aladdin; Shanghai; China) 
in $1 \mathrm{~L}$ normal BG11 medium. For theophylline-induced assays, Synechocystis samples were collected by centrifugation at $3000 \times g$ and $4{ }^{\circ} \mathrm{C}$ for $15 \mathrm{~min}$ and then re-suspended using fresh BG11 medium and corresponding amount of the stock solution of theophylline.

\section{SDS-PAGE}

SDS-PAGE was performed for WT, un-induced WTHMMA1(the $\left.{ }^{*}\right)$, and $2 \mathrm{mM}$ theophylline-induced WTHMMA1(the*). Briefly, samples (volume* $\mathrm{OD}_{730 \mathrm{~nm}}=5$ ) were collected by centrifugation at $7500 \times g$ and $4{ }^{\circ} \mathrm{C}$ for $10 \mathrm{~min}$. Then, the cell pellets were collected and resuspended using $100 \mu \mathrm{LddH} \mathrm{H}_{2} \mathrm{O}$. The samples were then crushed by multi-gelation with liquid nitrogen, and after another centrifugation $(14,000 \times g$ for $2 \mathrm{~min})$, supernatant was then used for SDS-PAGE. The total protein was quantified using the NanoDrop ${ }^{\mathrm{TM}} 2000 / 2000$ c spectro- $^{-}$ photometers (Thermo Fisher Scientific Inc., CA, USA), separated on $15 \%$ SDS-polyacrylamide gel and stained by coomassie blue $[57,58]$.

\section{Additional files}

Additional file 1: Figure S1. Schematic of the constructed vectors. Detailed sequences were available in Additional file 2: Table S2. A) Schematic of the pCP0168 and pBA0168. B) Schematic of the PBA3031M. Fig. S2. Validation of the expression of sRNAs in constructed strains using RT-PCR. A) The expression of sRNAs in WT-HMC, WT-PTGLGC, WT-HMGLGC, WT-HMFA, WT-HMMA1, WT-HMMA2, and WT-HMMA1 (the*). Lane 1-12: 1. WT-HMC using micC-R and RT-GlgC-F; 2. WT-PTGLGC using AsGlgc1-F and RT-PTGlgC-R; 3. WT-HMGLGC using micC-R and RT-GlgC-F; 4. WT-HMFA using micC-R and RT-sll1069-F; 5. WT-HMFA using micC-R and RT-slr1332F; 6. WT-HMFA using micC-R and RT-sIr1511-F; 7. WT-HMMA1 using micC-R and RT-slr1511-F; 8. WT-HMMA1 using micC-R and RT-slr2023-F; 9. WT-HMMA2 using micC-R and R RT-s\|l1069-F; 10. WT-HMMA2 using micC-R and RT-sIr1332-F; 11. WT-HMMA2 using micC-R and RT-slr1511-F; 12. WT-HMMA2 using micC-R and RT-sIr2023-F. B) The expression of sRNAs in WT-HMFA, WT-HMMA1, and WT-HMMA2. Lane 1-12: 1. WT-HMFA using micC-R and RT-SII1 069-F; 2. WT-HMFA using micC-R and RT-sIr1332-F; 3. WT-HMFA using micC-R and RT-sIr1511-F; 4. WT-HMMA1 using micC-R and RT-slr1511-F; 5. WT-HMMA1 using micC-R and RT-slr2023-F; 6. WTHMMA2 using micC-R and R RT-sll1069-F; 7. WT-HMMA2 using micC-R and RT-SIr1332-F; 8. WT-HMMA2 using micC-R and RT-sIr1511-F; 9. WT-HMMA2 using micC-R and RT-sIr2023-F; 10. HMFA using qRT-16S-F and qRT-16S-R; 11. HMMA1 using qRT-16S-F and qRT-16S-R; 12. HMMA2 using qRT-16S-F and gRT-16S-R. Fig. S3. Standard curve for glycogen measurement. A series of glucose standard diluents were utilized. Fig. S4. Standard curve for malonyl-CoA measurement. A series of malonyl-CoA standard diluents were utilized. Fig. S5. Bleaching phenotype of HMMA1 compared to WT. Fig. S6. A) Schematic of the theophylline-inducible riboswitch. B) Schematic of the pBA3031-HM(the*). Fig. S7. The dose effect of the theophylline on the growth of WT. The error bar represents the standard deviation of the three biological replicates for each sample. Fig. S8. SDSPAGE for WT and WT-HMMA1 (the*) with or without induction. Lane 1-7: 1 was WT: $2-4$ were WT-HMMA1 (the*) without induction and 5-7 were WT-HMMA1 (the*) with induction. The Hfa was shown using red arrows. Fig. S9. Binding energies distributions of all the genes of Synechocystis genome.

Additional file 2: Table S1. All the primers used in this study. Table S2. Detailed sequences for constructed plasmids in this study. Table S3. Sequences used for calculating binding energies between sRNAs and their corresponding targets.

\section{Abbreviations}

CRISPR/Cas system: clustered regularly interspaced short palindromic repeats/ CRISPR-associated proteins; FAMEs: fatty acid methyl esters; GC: gas chromatography; 3-HP: 3-hydroxypropionic acid; PTRNAs: paired termini RNAs; sRNA: small RNA; RBS: ribosome-binding site; WT: wild type.

\section{Authors' contributions}

TS performed the major experiments and wrote the draft manuscript; SBL, XYS, GSP, JJD, JYC, and MLS helped with some of the experiments; TS and WZ analyzed the data; TS, XYS, JJD, LC, and WZ designed and revised the manuscript. All authors read and approved the final manuscript.

\section{Author details \\ ${ }^{1}$ Laboratory of Synthetic Microbiology, School of Chemical Engineering \& Technology, Tianjin University, Tianjin 300072, People's Republic of China. ${ }^{2}$ Key Laboratory of Systems Bioengineering (Ministry of Education), Tianjin University, Tianjin 300072, People's Republic of China. ${ }^{3}$ SynBio Research Plat- form, Collaborative Innovation Center of Chemical Science and Engineering, Tianjin 300072, People's Republic of China. ${ }^{4}$ School of Environmental Science and Engineering, Tianjin University, Tianjin 300072, People's Republic of China. ${ }^{5}$ Center for Biosafety Research and Strategy, Tianjin University, Tianjin, People's Republic of China.}

\section{Acknowledgements}

We sincerely thank Prof. Song Xue and Dr. Jiao Liu from Dalian Institute of Chemical Physics, Chinese Academy of Sciences for the help with lipid profile analysis.

\section{Competing interests}

The authors declare that they have no competing interests.

\section{Availability of supporting data}

All data generated or analyzed during this study are included in this published article and its additional files.

\section{Consent for publication}

All authors agree to publication.

Ethics approval and consent to participate

Not applicable.

\section{Funding}

This work was supported by the National Science Foundation of China (NSFC) [Nos. 31770100, 21621004, 31370115, and 91751102], the National Basic Research Program of China (National "973" program) [No. 2014CB745101], and the National Science Foundation of Tianjin, China [No. 13JCQNJC09900].

\section{Publisher's Note}

Springer Nature remains neutral with regard to jurisdictional claims in published maps and institutional affiliations.

Received: 30 October 2017 Accepted: 23 January 2018

Published online: 05 February 2018

\section{References}

1. Clark JH, Luque R, Matharu AS. Green chemistry, biofuels, and biorefinery. Annu Rev Chem Biomol Eng. 2012;3:183-207.

2. Wirth TE, Gray CB, Podesta JD. The future of energy policy. Foreign Affairs. 2003;82:132-55.

3. Gao X, Sun T, Pei G, Chen L, Zhang W. Cyanobacterial chassis engineering for enhancing production of biofuels and chemicals. Appl Microbiol Biotechnol. 2016;100:3401-13.

4. Xu P, Gu Q, Wang W, Wong L, Bower AG, Collins CH, Koffas MA. Modular optimization of multi-gene pathways for fatty acids production in E. coli. Nat Commun. 2013;4:1409. 
5. Ruffing AM, Jensen TJ, Strickland LM. Genetic tools for advancement of Synechococcus sp. PCC 7002 as a cyanobacterial chassis. Microb Cell Fact. 2016;15:190.

6. Ungerer J, Pakrasi HB. Cpf1 is a versatile tool for CRISPR genome editing across diverse species of cyanobacteria. Sci Rep. 2016;6:39681.

7. Mandal M, Breaker RR. Gene regulation by riboswitches. Nat Rev Mol Cell Biol. 2004;5:451-63.

8. Salis HM, Mirsky EA, Voigt CA. Automated design of synthetic ribosome binding sites to control protein expression. Nat Biotechnol. 2009;27:946-50

9. Jiang W, Bikard D, Cox D, Zhang F, Marraffini LA. CRISPR-assisted editing of bacterial genomes. Nat Biotechnol. 2013;31:233-9.

10. Na D, Yoo SM, Chung H, Park H, Park JH, Lee SY. Metabolic engineering of Escherichia coli using synthetic small regulatory RNAs. Nat Biotechnol. 2013:31:170-4.

11. Kang Z, Zhang C, Zhang J, Jin P, Zhang J, Du G, Chen J. Small RNA regulators in bacteria: powerful tools for metabolic engineering and synthetic biology. Appl Microbiol Biotechnol. 2014:98:3413-24.

12. Gaida SM, Al-Hinai MA, Indurthi DC, Nicolaou SA, Papoutsakis ET. Synthetic tolerance: three noncoding small RNAs, DsrA, ArcZ and RprA, acting supra-additively against acid stress. Nucleic Acids Res. 2013:41:8726-37.

13. Rodrigo G, Landrain TE, Jaramillo A. De novo automated design of small RNA circuits for engineering synthetic riboregulation in living cells. Proc Natl Acad Sci USA. 2012;109:15271-6.

14. Green AA, Silver PA, Collins JJ, Yin P. Toehold switches: de-novo-designed regulators of gene expression. Cell. 2014;159:925-39.

15. Nakashima N, Tamura T. Conditional gene silencing of multiple genes with antisense RNAs and generation of a mutator strain of Escherichia coli. Nucleic Acids Res. 2009;37:e103.

16. Nakashima N, Tamura T, Good L. Paired termini stabilize antisense RNAs and enhance conditional gene silencing in Escherichia coli. Nucleic Acids Res. 2006;34:e138,

17. Wu J, Yu O, Du G, Zhou J, Chen J. Fine-tuning of the fatty acid pathway by synthetic antisense RNA for enhanced (2S)-naringenin production from L-tyrosine in Escherichia coli. Appl Environ Microbiol. 2014;80:7283-92.

18. Yang Y, Lin Y, Li L, Linhardt RJ, Yan Y. Regulating malonyl-CoA metabolism via synthetic antisense RNAs for enhanced biosynthesis of natural products. Metab Eng. 2015;29:217-26.

19. Argaman L, Elgrably-Weiss M, Hershko T, Vogel J, Altuvia S. RelA protein stimulates the activity of RyhB small RNA by acting on RNA-binding protein Hfa. Proc Natl Acad Sci USA. 2012;109:4621-6.

20. Aiba H. Mechanism of RNA silencing by Hfq-binding small RNAs. Curr Opin Microbiol. 2007:10:134-9.

21. Moll I, Afonyushkin T, Vytvytska O, Kaberdin VR, Blasi U. Coincident Hfa binding and RNase E cleavage sites on mRNA and small regulatory RNAs. RNA. 2003;9:1308-14.

22. Cho C, Lee SY. Efficient gene knockdown in Clostridium acetobutylicum by synthetic small regulatory RNAs. Biotechnol Bioeng. 2017;114:374-83.

23. Kopf M, Hess WR. Regulatory RNAs in photosynthetic cyanobacteria. FEMS Microbiol Rev. 2015;39:301-15.

24. Sun T, Pei G, Wang J, Chen L, Zhang W. A novel small RNA CoaR regulates coenzyme A biosynthesis and tolerance of Synechocystis sp. PCC6803 to 1-butanol possibly via promoter-directed transcriptional silencing. Biotechnol Biofuels. 2017;10:42

25. Hu J, Li T, Xu W, Zhan J, Chen H, He C, Wang Q. Small antisense RNA RbIR positively regulates RuBisCo in Synechocystis sp. PCC 6803. Front Microbiol. 2017:8:231

26. Zess EK, Begemann MB, Pfleger BF. Construction of new synthetic biology tools for the control of gene expression in the cyanobacterium Synechococcus sp. strain PCC 7002. Biotechnol Bioeng. 2016;113:424-32.

27. Tan X, Yao L, Gao Q, Wang W, Qi F, Lu X. Photosynthesis driven conversion of carbon dioxide to fatty alcohols and hydrocarbons in cyanobacteria. Metab Eng. 2011;13:169-76.

28. Zhou J, Zhang H, Meng H, Zhu Y, Bao G, Zhang Y, Li Y, Ma Y. Discovery of a super-strong promoter enables efficient production of heterologous proteins in cyanobacteria. Sci Rep. 2014;4:4500.

29. Mohamed A, Eriksson J, Osiewacz HD, Jansson C. Differential expression of the psbA genes in the cyanobacterium Synechocystis 6803. Mol Gen Genet. 1993;238:161-8.
30. Yao L, Cengic I, Anfelt J, Hudson EP. Multiple gene repression in cyanobacteria using CRISPRi. ACS Synth Biol. 2016;5:207-12.

31. Ponchon L, Catala M, Seijo B, El Khouri M, Dardel F, Nonin-Lecomte S, Tisne C. Co-expression of RNA-protein complexes in Escherichia coli and applications to RNA biology. Nucleic Acids Res. 2013;41:e150.

32. Grundel M, Scheunemann R, Lockau W, Zilliges Y. Impaired glycogen synthesis causes metabolic overflow reactions and affects stress responses in the cyanobacterium Synechocystis sp. PCC 6803. Microbiology. 2012;158:3032-43.

33. Cronan JE, Thomas J. Bacterial fatty acid synthesis and its relationships with polyketide synthetic pathways. Methods Enzymol. 2009:459:395-433.

34. Wang Y, Sun T, Gao X, Shi M, Wu L, Chen L, Zhang W. Biosynthesis of platform chemical 3-hydroxypropionic acid (3-HP) directly from $\mathrm{CO}_{2}$ in cyanobacterium Synechocystis sp. PCC 6803. Metab Eng. 2016;34:60-70.

35. Nakahira Y, Ogawa A, Asano H, Oyama T, Tozawa Y. Theophylline-dependent riboswitch as a novel genetic tool for strict regulation of protein expression in Cyanobacterium Synechococcus elongatus PCC 7942. Plant Cell Physiol. 2013;54:1724-35.

36. Ohbayashi R, Akai H, Yoshikawa H, Hess WR, Watanabe S. A tightly inducible riboswitch system in Synechocystis sp. PCC 6803. J Gen Appl Microbiol. 2016:62:154-9.

37. Wright PR, Georg J, Mann M, Sorescu DA, Richter AS, Lott S, Kleinkauf R, Hess WR, Backofen R. CopraRNA and IntaRNA: predicting small RNA targets, networks and interaction domains. Nucleic Acids Res. 2014;42:W119-23.

38. Mann M, Wright PR, Backofen R. IntaRNA 2.0: enhanced and customizable prediction of RNA-RNA interactions. Nucleic Acids Res. 2017. https://doi. org/10.1093/nar/gkx1279.

39. Oliver JW, Machado IM, Yoneda H, Atsumi S. Combinatorial optimization of cyanobacterial 2,3-butanediol production. Metab Eng. 2014;22:76-82.

40. Gordon GC, Korosh TC, Cameron JC, Markley AL, Begemann MB, Pfleger BF. CRISPR interference as a titratable, trans-acting regulatory tool for metabolic engineering in the cyanobacterium Synechococcus sp. strain PCC 7002. Metab Eng. 2016;38:170-9.

41. Beld J, Abbriano R, Finzel K, Hildebrand M, Burkart MD. Probing fatty acid metabolism in bacteria, cyanobacteria, green microalgae and diatoms with natural and unnatural fatty acids. Mol BioSyst. 2016;12:1299-312.

42. Kuo J, Khosla C. The initiation ketosynthase (FabH) is the sole rate-limiting enzyme of the fatty acid synthase of Synechococcus sp. PCC 7002. Metab Eng. 2014;22:53-9.

43. Parsons JB, Rock CO. Bacterial lipids: metabolism and membrane homeostasis. Prog Lipid Res. 2013;52:249-76.

44. Noh M, Yoo SM, Kim WJ, Lee SY. Gene expression knockdown by modulating synthetic small RNA expression in Escherichia coli. Cell Syst. 2017. https://doi.org/10.1016/j.cels.2017.1008.1016.

45. Shi S, Chen Y, Siewers V, Nielsen J. Improving production of malonyl coenzyme A-derived metabolites by abolishing Snf1-dependent regulation of Acc1. MBio. 2014:5:e01130-01114.

46. Liu C, Ding Y, Xian M, Liu M, Liu H, Ma Q, Zhao G. Malonyl-CoA pathway: a promising route for 3-hydroxypropionate biosynthesis. Crit Rev Biotechnol. 2017. https://doi.org/10.1080/07388551.2016.1272093.

47. Chen X, Yang X, Shen Y, Hou J, Bao X. Increasing malonyl-CoA derived product through controlling the transcription regulators of phospholipid synthesis in Saccharomyces cerevisiae. ACS Synth Biol. 2017:6:905-12.

48. Lynch SA, Gallivan JP. A flow cytometry-based screen for synthetic riboswitches. Nucleic Acids Res. 2009:37:184-92.

49. Topp S, Reynoso CM, Seeliger JC, Goldlust IS, Desai SK, Murat D, Shen A, Puri AW, Komeili A, Bertozzi CR, et al. Synthetic riboswitches that induce gene expression in diverse bacterial species. Appl Environ Microbiol. 2010;76:7881-4.

50. Wachsmuth M, Findeiss S, Weissheimer N, Stadler PF, Morl M. De novo design of a synthetic riboswitch that regulates transcription termination. Nucleic Acids Res. 2013:41:2541-51.

51. Liu J, Chen L, Wang J, Qiao J, Zhang W. Proteomic analysis reveals resistance mechanism against biofuel hexane in Synechocystis sp. PCC 6803. Biotechnol Biofuels. 2012;5:68.

52. Fang F, Barnum SR. Expression of the heat shock gene hsp16.6 and promoter analysis in the cyanobacterium, Synechocystis sp. PCC 6803. Curr Microbiol. 2004:49:192-8. 
53. Kopka J, Schmidt S, Dethloff F, Pade N, Berendt S, Schottkowski M, Martin N, Duhring U, Kuchmina E, Enke H, et al. Systems analysis of ethanol production in the genetically engineered cyanobacterium Synechococcus sp. PCC 7002. Biotechnol Biofuels. 2017;10:56.

54. Huang S, Chen L, Te R, Qiao J, Wang J, Zhang W. Complementary iTRAQ proteomics and RNA-seq transcriptomics reveal multiple levels of regulation in response to nitrogen starvation in Synechocystis sp. PCC 6803. Mol BioSyst. 2013;9:2565-74.

55. Livak KJ, Schmittgen TD. Analysis of relative gene expression data using real-time quantitative PCR and the 2(-Delta Delta C(T)) method. Methods. 2001;25:402-8.
56. Liu J, Liu Y, Wang H, Xue S. Direct transesterification of fresh microalgal cells. Bioresour Technol. 2015;176:284-7.

57. Bradford MM. A rapid and sensitive method for the quantitation of microgram quantities of protein utilizing the principle of protein-dye binding. Anal Biochem. 1976;72:248-54.

58. Laemmli UK. Cleavage of Structural Proteins during the Assembly of the Head of Bacteriophage T4. Nature. 1970;227:680.

\section{Submit your next manuscript to BioMed Central and we will help you at every step:}

- We accept pre-submission inquiries

- Our selector tool helps you to find the most relevant journal

- We provide round the clock customer support

- Convenient online submission

- Thorough peer review

- Inclusion in PubMed and all major indexing services

- Maximum visibility for your research

Submit your manuscript at www.biomedcentral.com/submit 